\title{
Regulation of leucoplast morphology in roots
}

\author{
Interorganellar signaling from mitochondria?
}

\begin{abstract}
Ryuuichi D. Itoh ${ }^{1, *}$ and Makoto T. Fujiwara ${ }^{2}$
${ }^{1}$ Department of Chemistry, Biology and Marine Science; Faculty of Science; University of the Ryukyus; Nishihara, Okinawa Japan;

${ }^{2}$ Department of Life Sciences; Graduate School of Arts and Sciences; University of Tokyo; Komaba, Tokyo Japan
\end{abstract}

Key words: antimycin A, interorganellar crosstalk, plastid morphology, respiration, stress response, stromule

Submitted: 03/23/10

Accepted: 03/23/10

Previously published online: www.landesbioscience.com/journals/psb/ article/11893

*Correspondence to: Ryuuichi D. Itoh; Email: ryuitoh@sci.u-ryukyu.ac.jp

Addendum to: Itoh RD, Yamasaki H, Septiana

A, Yoshida S, Fujiwara MT. Chemical in-

duction of rapid and reversible plastid

filamentation in Arabidopsis thaliana roots.

Physiol Plant 2010; PMID: 20088905; DOI:

10.1111/j.1399-3054.2010.01352.x.
$\mathrm{T}$ he appearance of leaf mesophyll chloroplasts in angiosperms is characterized by their uniform and static shape, which is molded by symmetric division of the preexisting organelles, involving three prokaryote-derived proteins: the division executor protein, FtsZ, and the division site positioning proteins, MinD and MinE. On the other hand, noncolored plastids in roots, where the involvement of the known chloroplast division factors in plastid morphogenesis is yet unclear, are morphologically heterogeneous and transform dynamically. This is further emphasized by the active formation of long tubular protrusions called stromules from the main body of those plastids. Molecular regulation and physiological significance of such dynamic morphology of root plastids also remain unknown. In this context, we have recently demonstrated that the mitochondrial respiratory inhibitor antimycin A induces rapid and reversible filamentation of root plastids (leucoplasts) in Arabidopsis thaliana. In contrast, the same treatment with antimycin A did not affect the morphology of amyloplasts in the columella cells at the root tip. The alternative oxidase inhibitor salicylhydroxamic acid suppresses the antimycininduced plastid filamentation, perhaps implying an alternative oxidase-mediated interorganellar signaling between the mitochondria and the leucoplasts in the root cells. Our data may provide some clues as to how the formation of stromules is initiated.

\section{Plasticity of Plastid Morphology in Roots}

The morphology of chloroplastschlorophyll-containing photosynthetic plastids - in the leaf mesophyll cells in higher plants is mainly characterized by their uniform and simple shape, which is usually spherical or oval. The pioneering discovery of a chloroplast division mutant of Arabidopsis thaliana, named arc11 (accumulation and replication of chloroplasts 11), which exhibits a heterogeneous population of mesophyll chloroplasts caused by their asymmetric division, ${ }^{1}$ revealed that there must be proteinaceous factors that specify the symmetric chloroplast division to generate two equalsized daughter chloroplasts. Subsequent studies have shown that several proteins contribute to the symmetry of chloroplast division by confining the assembly of the PD/dynamin/FtsZ (PDF) triadic division ring within the mid-chloroplast region. The division ring-positioning factors in vascular plants include prokaryote-derived stromal proteins $\mathrm{MinD}$ and MinE, which antagonistically interact with each other to allow FtsZ (also known as a prokaryote-derived stromal protein) to form a ring exclusively in the mid-chloroplast, ${ }^{2}$ and chloroplast-specific proteins ARC3, MCD1 and PARC6 (also referred to as CDP1 or ARC6H), which were later invented and integrated into the positioning system at different times (in that order) during the course of land plant evolution. ${ }^{3}$ 

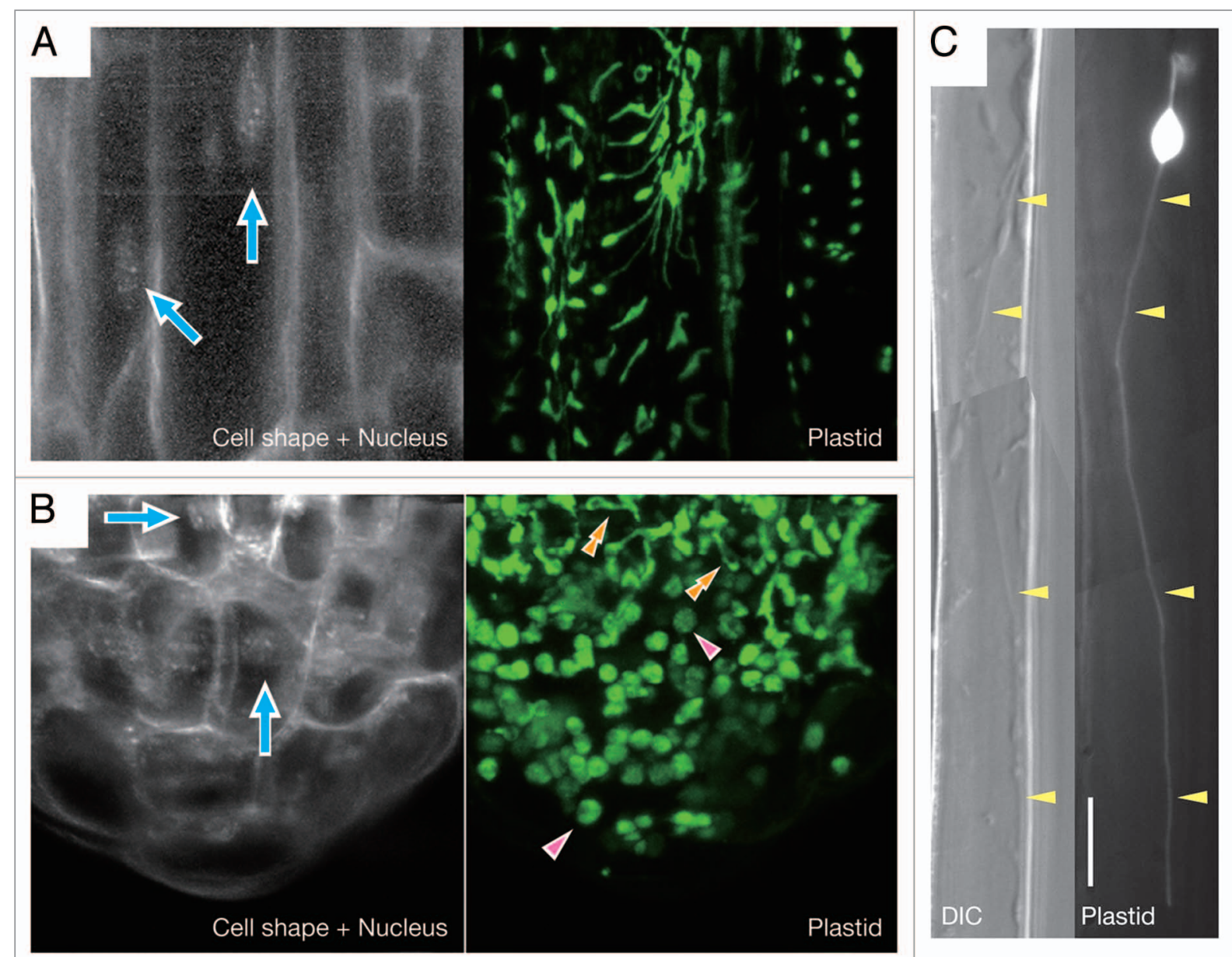

Figure 1. Morphology of non-green plastids in Arabidopsis thaliana roots. Plastids in the epidermal cells of main roots (A and C) and the root tip, including root cap and meristem (B), were visualized by plastid-targeted green fluorescence protein, pt-sGFP(S65T). ${ }^{15}$ For each panel, GFP images are shown at the right. For (A and B), 4',6-diamidino-2-phenylindole (DAPI)-stained fluorescent image of the same field of view as the GFP image is at the left to show the positions of cell nuclei (indicated by arrows) and the cell outlines. In (B), single arrowheads and double arrowheads indicate mature amyloplasts in the columella cells and transitional plastids that are differentiating into amyloplasts from proplastids, respectively. For (C), the differential interference contrast (DIC) image of the same field of view as the GFP image is at the left to show the presence of an exceptionally long stromule (indicated by arrowheads), which reaches a length of about $96.8 \mu \mathrm{m}$ including the length of the plastid main body. Bar $=10 \mu \mathrm{m}$.

In contrast to leaf mesophyll chloroplasts, non-green plastids in the root cells, which are usually categorized as leucoplasts or amyloplasts depending on the degree of their starch accumulation, are highly heterogeneous in their shape and size and also transform dynamically, as revealed by the visualization of those noncolored organelles by plastid-targeted fluorescence proteins $^{4}$ (Fig. 1). The most conspicuous feature of the plastid morphology in roots is the exuberant formation of stromules, the stroma-containing, double envelopebound tubules, which themselves also actively transform ${ }^{5,6}$ (Fig. 1). Recent studies have suggested the regulation of plastid morphology by some chloroplast division factors, such as ARC3, ARC6, ${ }^{7} \mathrm{MinE}^{8}$ and
FtsZ (our unpublished observations), even in the root cells, maybe through the process of plastid replication. Nevertheless, the detailed mechanism of this morphological regulation on the root plastids remains totally unknown. Furthermore, it seems obvious that most of morphological alterations of plastids occur without associated replication of the organelles. Then, what exactly governs the morphology of the root plastids as well as its maintenance and dynamic change?

\section{Effects of Antimycin A on Plastid Morphology in Roots}

Our recent report may provide a hint about the above question. ${ }^{9}$ We found that antimycin $A$, an effective inhibitor of respiratory electron transport in the mitochondria, induces extreme filamentation of plastids (leucoplasts) in the main roots of $A$. thaliana, preferentially in the mature cortex (Fig. 2A and B). The plastid filamentation is rapid, being initiated within 5 minutes after the onset of antimycin treatment and completed within 1 hour. In addition, the filamentation is a reversible response: morphology of the plastids was fully restored within 7 hours after the washout of antimycin. As expected from its rapid initiation, the antimycin-induced plastid filamentation was not suppressed by the protein synthesis inhibitors, cycloheximide and chloramphenicol, implying that this response is not mediated by de 
novo protein synthesis in the cytoplasm and organelles. Similarly, the cytoskeletal inhibitors, demecolcine and latrunculin B, did not suppress the plastid filamentation under our experimental condition, although there are several lines of evidence for the interaction between stromules and the cytoskeleton. ${ }^{6,10-12}$

In spite of the powerful and broadranging effect of antimycin $\mathrm{A}$ on the morphology of leucoplasts in the mature root cortex, we could not detect major differences in the morphology of plastids in the hypocotyls, cotyledon epidermis, and true leaf epidermis and mesophylls between the antimycin-treated seedlings and the mock-treated control.' In this study, we have extended our observation of plastids to the columella cells at the root tip, which contain well-developed amyloplasts, and found no significant differences again in the amyloplast morphology therein between inhibitor- and mock-treated plants (Fig. 2C and D). One may question why the antimycin's effect appears to be specific to root cortex plastids. Although we have no answer for this question at the moment, it might be worth noting that among those we used for the inhibitor experiment, the root cortex is the only tissue in which active formation of stromules is constantly observed as a normal state.

Among the chemicals we used for the co-application with antimycin A, salicylhydroxamic acid (SHAM) was the only one that suppressed the plastid filamentation.' SHAM is known as a typical inhibitor of alternative oxidase (AOX). AOX is a cyanide-resistant mitochondrial enzyme, which allows electrons on the respiratory chain to bypass cytochrome $b c$ complex and cytochrome $c$ oxidase and thus to reduce the oxygen when cytochrome-mediated electron transport is blocked by chemicals, such as cyanide or antimycin A, or by certain stress conditions, such as wounding of tissue or chilling. ${ }^{13}$ Because AOX is thought to be activated by blockage of the cytochrome pathway, we proposed the hypothesis that the plastid filamentation upon antimycin treatment might be caused by some sort of an interorganellar signal, which would be derived from the activated AOX in the mitochondria. This idea would connect stress conditions surrounding the plants to

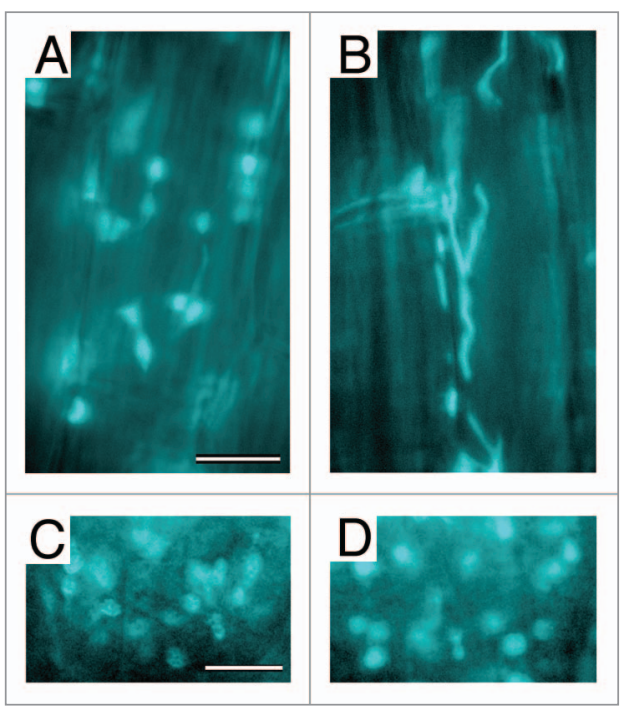

Figure 2. Effects of antimycin A on plastid morphology in Arabidopsis thaliana roots. Leucoplasts in the mature cortex of main roots ( $A$ and B) and amyloplasts in the columella cells at the root tip ( $C$ and $D$ ) from 1-week-old seedlings were visualized by plastid-targeted cyan fluorescence protein (CFP). CFP images are shown for the mock-treated sample ( $A$ and $C$ ) and for the samples treated for 2 hours with $50 \mu \mathrm{M}$ antimycin $\mathrm{A}$ (B and D). Bar $=10 \mu \mathrm{m}$.

morphological changes of their plastids through dysfunction of the cytochrome pathway of mitochondrial electron transport, activation of $A O X$, and generation of AOX-derived signal directed to the plastids, although the identity, if any, of this hypothetical signal remains entirely an open question at present. While we found no obvious differences in plastid morphology in the hypocotyls, cotyledon epidermis, and true leaf epidermis and mesophylls between antimycin-treated and nontreated Arabidopsis thaliana, ${ }^{9}$ high temperature-induced formation of chloroplast protrusions and stromules was previously observed in the leaf mesophylls. ${ }^{14}$ Undoubtedly, the tissue specificity and physiological relevance of plastid filamentation, as well as stromule formation, are the important issues to be clarified, which deserve further investigation in the future.

\section{Acknowledgements}

We thank Dr. Yasuo Niwa (Univ. of Shizuoka) for the gift of the GFP transgenic line. This research was supported by Grants-in-Aid from the Ministry of Education, Culture, Sports, Science and Technology (MEXT) of Japan (20657015 to R.D.I.) and the Agricultural Chemical Research Foundation (to M.T.F. and R.D.I.).

\section{References}

1. Marrison JL, Rutherford SM, Robertson EJ, Lister C, Dean C, Leech RM. The distinctive roles of five different $A R C$ genes in the chloroplast division process in Arabidopsis. Plant J 1999; 18:651-62.

2. Fujiwara MT, Hashimoto H, Kazama Y, Abe T, Yoshida S, Sato N, Itoh RD. The assembly of the FtsZ ring at the mid-chloroplast division site depends on a balance between the activities of AtMinE1 and ARC11/AtMinD1. Plant Cell Physiol 2008; 49:345-61.

3. Okazaki K, Kabeya Y, Miyagishima S. The evolution of the regulatory mechanism of chloroplast division. Plant Signal Behav 2010; 5.

4. Köhler RH, Schwille P, Webb WW, Hanson MR. Active protein transport through plastid tubules: velocity quantified by fluorescence correlation spectroscopy. J Cell Sci 2000; 113:3921-30.

5. Köhler RH, Cao J, Zipfel WR, Webb WW, Hanson MR. Exchange of protein molecules through connections between higher plant plastids. Science 1997; 276:2039-42.

6. Gunning BE. Plastid stromules: video microscopy of their outgrowth, retraction, tensioning, anchoring, branching, bridging and tip-shedding. Protoplasma 2005; 225:33-42.

7. Holzinger A, Kwok EY, Hanson MR. Effects of arc3, $\operatorname{arc} 5$ and $\operatorname{arc} 6$ mutations on plastid morphology and stromule formation in green and nongreen tissues of Arabidopsis thaliana. Photochem Photobiol 2008; 84:1324-35.

8. Kojo KH, Fujiwara MT, Itoh RD. Involvement of AtMinE1 in plastid morphogenesis in various tissues of Arabidopsis thaliana. Biosci Biotechnol Biochem 2009; 73:2632-9.

9. Itoh RD, Yamasaki H, Septiana A, Yoshida S, Fujiwara MT. Chemical induction of rapid and reversible plastid filamentation in Arabidopsis thaliana roots. Physiol Plant 2010; DOI: 10.1111/j.13993054.2010.01352.x

10. Kwok EY, Hanson MR. Microfilaments and microtubules control the morphology and movement of nongreen plastids and stromules in Nicotiana tabacum. Plant J 2003; 35:16-26. 
11. Kwok EY, Hanson MR. In vivo analysis of interactions between GFP-labeled microfilaments and plastid stromules. BMC Plant Biol 2004; 4:2.

12. Natesan SKA, Sullivan JA, Gray JC. Myosin XI is required for actin-associated movement of plastid stromules. Mol Plant 2009; 2:1262-72.

13. Wagner AM, Klaas K. The alternative respiration pathway in plants: Role and regulation. Physiol Plant $1995 ; 95: 318-25$
14. Holzinger A, Buchner O, Lütz C, Hanson MR Temperature-sensitive formation of chloroplast protrusions and stromules in mesophyll cells of Arabidopsis thaliana. Protoplasma 2007; 230:23-30.

15. Niwa Y, Hirano T, Yoshimoto K, Shimizu M, Kobayashi H. Non-invasive quantitative detection and applications of non-toxic, S65T-type green fluorescent protein in living plants. Plant J 1999; $18: 455-63$ 